\title{
Commutation-relation-preserving ladder operators for propagating optical fields in nonuniform lossy media
}

Partanen, Mikko; Häyrynen, Teppo; Tulkki, Jukka; Oksanen, Jani

Published in:

Physical Review A

Link to article, DOI:

10.1103/physreva.92.033839

Publication date:

2015

Document Version

Publisher's PDF, also known as Version of record

Link back to DTU Orbit

Citation (APA):

Partanen, M., Häyrynen, T., Tulkki, J., \& Oksanen, J. (2015). Commutation-relation-preserving ladder operators for propagating optical fields in nonuniform lossy media. Physical Review A, 92(3), [033839].

https://doi.org/10.1103/physreva.92.033839

\section{General rights}

Copyright and moral rights for the publications made accessible in the public portal are retained by the authors and/or other copyright owners and it is a condition of accessing publications that users recognise and abide by the legal requirements associated with these rights.

- Users may download and print one copy of any publication from the public portal for the purpose of private study or research.

- You may not further distribute the material or use it for any profit-making activity or commercial gain

- You may freely distribute the URL identifying the publication in the public portal 


\title{
Commutation-relation-preserving ladder operators for propagating optical fields in nonuniform lossy media
}

\author{
Mikko Partanen, ${ }^{1}$ Teppo Häyrynen, ${ }^{1,2}$ Jukka Tulkki, ${ }^{1}$ and Jani Oksanen ${ }^{1}$ \\ ${ }^{1}$ Engineered Nanosystems group, School of Science, Aalto University, P.O. Box 12200, 00076 Aalto, Finland \\ ${ }^{2}$ DTU Fotonik, Department of Photonics Engineering, Technical University of Denmark, Ørsteds Plads, Building 343, \\ DK-2800 Kongens Lyngby, Denmark \\ (Received 3 June 2015; published 21 September 2015)
}

\begin{abstract}
We have recently developed a quantized fluctuational electrodynamics (QFED) formalism to describe the quantum aspects of local thermal balance formation and to formulate the electromagnetic field ladder operators so that they no longer exhibit the anomalies reported for resonant structures. Here we show how the QFED can be used to resolve between the left and right propagating fields to bridge the QFED and the quantum optical input-output relations commonly used to describe selected quantum aspects of resonators. The generalized model introduces a density of states concept describing interference effects, which is instrumental in allowing an unambiguous separation of the fields and related quantum operators into left and right propagating parts. In addition to providing insight on the quantum treatment of interference, our results also provide the conclusive resolution of the long-standing enigma of the anomalous commutation relations of partially confined propagating fields.
\end{abstract}

DOI: 10.1103/PhysRevA.92.033839

PACS number(s): 42.50.Nn, 42.50.Ct, 42.50.Gy, 42.50.Lc

\section{INTRODUCTION}

It has recently been suggested [1-4] that in contrast to earlier predictions the commutation relations of photon ladder operators have directly measurable physical significance. This is especially interesting in the case of resonant structures where conventional theoretical descriptions have been shown to lead to anomalous commutation relations of the general form $\left[\hat{a}, \hat{a}^{\dagger}\right]=\Lambda \neq 1$, instead of the canonical form with $\Lambda=1$ [5-9]. The anomaly is a direct consequence of the conventional piecewise normalization of the optical modes in respective homogeneous regions and of the interference effects coupling the modes propagating in different directions. Originally these anomalies were argued to bear no physical significance, but recently it has been shown that the anomalous commutation relations lead to the existence of a threshold for second harmonic generation when it occurs inside microcavities $[1,2]$. In addition, anomalies in the commutation relations have also been shown to prevent systematic description of the local thermal balance between the field and interacting media $[3,4,10]$. Experimental measurements of the onset of the second harmonic generation or the thermal balance formation in microcavities could therefore confirm the theoretical predictions that the conventional mode normalization introducing these anomalies is not sufficient for field quantization of resonant structures. Here, we develop a field quantization approach that (1) fully eliminates the anomalies for propagating fields, (2) bridges the classical propagating wave and commutation-relation-preserving quantum descriptions, and (3) allows formulating conceptually simple models for optical energy transfer and the formation of thermal balance in interfering nanostructures.

One of the most widely used quantization approaches for describing spatial field evolution in resonant structures is the input-output relation formalism of the photon creation and annihilation operators. The formalism was originally developed for dispersionless and lossless media [11] and later extended for lossy and dispersive dielectrics by several groups [12-17]. The quantization procedures studied, e.g., by Barnett et al. [15] clearly highlight that the noise and field operators in nonuniform systems are position dependent and that the vector potential and electric-field operators obey the well-known canonical commutation relation as expected $[15,16]$. The canonical commutation relations in these early models did not, however, extend to the ladder operators which were found to exhibit anomalies in resonant structures [5]. The anomalous commutation relations of the ladder operators were later studied in several works [6-9] but no clear resolution for the anomalies was found. Instead it was concluded that the anomalies as well as the exact form of the ladder operators within resonant structures were irrelevant as long as the field commutation relations and classical field quantities were well defined. However, this made it impossible to fully quantize the optical fields in resonant structures.

To shed more light on the anomalous commutation relations, we have very recently developed a quantized fluctuational electrodynamics (QFED) scheme based on generalizing the fluctuational electrodynamics to quantum optical fields $[3,4,10]$. Using the QFED approach we were able to formulate the canonical commutation relations preserving ladder and photon-number operators for the total electromagnetic (EM) field $[3,4,10]$. However, even in the QFED framework, it has not been evident how to separate the ladder and photon-number operators to left and right propagating parts, which is also essential for the final resolution of the anomalies and for bridging the classical propagating wave descriptions and the commutation-relation-preserving quantum descriptions. In this work, we show that the QFED can be extended to resolve between the left and right propagating fields, fully preserving the canonical commutations with $\Lambda=1$ also for the left and right propagating field ladder operators. As it turns out, the separation to the left and right propagating fields becomes possible and conceptually simple when one introduces a new density of states concept describing the fundamentally important interference effects. The added insight obtained using the introduced concepts provides a more detailed understanding 
of the quantization of optical fields in complex surroundings, and can be used, e.g., for finding simple photon-number-based expressions for the quantum optical Poynting vector as well as more detailed description of quantized energy flow in resonant structures.

\section{FIELD QUANTIZATION}

\section{A. Photon operators}

In contrast to previous approaches, the requirement of the QFED is the preservation of the local canonical commutation relation $\left[\hat{a}(x, \omega), \hat{a}^{\dagger}\left(x, \omega^{\prime}\right)\right]=\delta\left(\omega-\omega^{\prime}\right)$ also in resonant media $[3,10]$. This requirement leads to conceptually simple definitions for the position-dependent ladder and photon-number operators as a weighted sum over the incident fields and the noise. For the expectation value of the photon-number operator, for instance, this weighted sum reads as [4]

$$
\langle\hat{n}(x, \omega)\rangle=\frac{\int_{-\infty}^{\infty} \rho_{\mathrm{NL}}\left(x, \omega, x^{\prime}\right)\left\langle\hat{\eta}\left(x^{\prime}, \omega\right)\right\rangle d x^{\prime}}{\int_{-\infty}^{\infty} \rho_{\mathrm{NL}}\left(x, \omega, x^{\prime}\right) d x^{\prime}},
$$

where $\langle\hat{\eta}(x, \omega)\rangle$ is the source field photon-number expectation value which for thermal fields is given by the BoseEinstein distribution $\left\langle\hat{\eta}\left(x^{\prime}, \omega\right)\right\rangle=1 /\left[e^{\hbar \omega /\left[k_{\mathrm{B}} T\left(x^{\prime}\right)\right]}-1\right]$ with the position-dependent temperature of the medium given by $T\left(x^{\prime}\right)$. The weighting coefficient $\rho_{\mathrm{NL}}\left(x, \omega, x^{\prime}\right)$ in Eq. (1) is given by

$$
\begin{aligned}
\rho_{\mathrm{NL}}\left(x, \omega, x^{\prime}\right)= & \frac{\omega^{3}|\varepsilon(x, \omega)|}{\pi c^{4} S} \varepsilon_{\mathrm{i}}\left(x^{\prime}, \omega\right) \\
& \times\left(\left|G\left(x, \omega, x^{\prime}\right)\right|^{2}+\left|\frac{\partial G\left(x, \omega, x^{\prime}\right)}{k(x, \omega) \partial x}\right|^{2}\right),
\end{aligned}
$$

where $c$ is the speed of light in vacuum, $S$ is the area of quantization in the $y-z$ plane, $\varepsilon(x, \omega)=n(x, \omega)^{2}$ is the relative electric permittivity of a nonmagnetic medium with refractive index $n(x, \omega)$ and $\varepsilon_{\mathrm{i}}(x, \omega)$ is its imaginary part, $k(x, \omega)=\omega n(x, \omega) / c$ is the wave number, and $G\left(x, \omega, x^{\prime}\right)$ is the Green's function of the Helmholtz equation given for selected layered structures in Ref. [3]. The quantity $\rho_{\mathrm{NL}}\left(x, \omega, x^{\prime}\right)$ is here referred to as the nonlocal density of states (NLDOS) since it highlights the nonlocal origin of the local density of states $($ LDOS) $\rho(x, \omega)$ given as

$$
\rho(x, \omega)=\int_{-\infty}^{\infty} \rho_{\mathrm{NL}}\left(x, \omega, x^{\prime}\right) d x^{\prime},
$$

and appearing in the denominator of Eq. (1). After integration, the LDOS can also be expressed in the more familiar form in terms of the imaginary part of the Green's function [4]. Using this definition, the NLDOS $\rho_{\mathrm{NL}}\left(x, \omega, x^{\prime}\right)$ accounts for both the electric-field (term $|G|^{2}$ ) and the magnetic-field (term $\left.|\partial G /(k \partial x)|^{2}\right)$ contributions.

\section{B. Quantum optical Poynting vector}

The quantum optical Poynting vector $\hat{S}(x, t)$ that will be used as a starting point for separating the field components is defined in terms of the positive $(+)$ and negative $(-)$ frequency parts of the electric- and magnetic-field operators $\hat{E}(x, t)$ and $\hat{B}(x, t)$ as $\hat{S}(x, t)=\varepsilon_{0} c^{2}\left[\hat{E}^{-}(x, t) \hat{B}^{+}(x, t)+\hat{B}^{-}(x, t) \hat{E}^{+}(x, t)\right]$ $[18,19]$. Using the QFED framework and substituting the electric- and magnetic-field operators as given in Ref. [3], we write the Poynting vector expectation value at angular frequency $\omega$ as

$$
\langle\hat{S}(x, t)\rangle_{\omega}=\hbar \omega v(x, \omega) \int_{-\infty}^{\infty} \rho_{\mathrm{IF}}\left(x, \omega, x^{\prime}\right)\left\langle\hat{\eta}\left(x^{\prime}, \omega\right)\right\rangle d x^{\prime},
$$

where $v(x, \omega)=c / n_{\mathrm{r}}(x, \omega)$ is the energy propagation velocity, $n_{\mathrm{r}}(x, \omega)$ is the real part of the refractive index $n(x, \omega)=$ $\sqrt{\varepsilon(x, \omega)}$, and

$$
\begin{aligned}
\rho_{\mathrm{IF}}\left(x, \omega, x^{\prime}\right)= & \frac{2 \omega n_{\mathrm{r}}(x, \omega)}{\pi c^{3} S} \varepsilon_{\mathrm{i}}\left(x^{\prime}, \omega\right) \\
& \times \operatorname{Re}\left(i \omega G\left(x, \omega, x^{\prime}\right) \frac{\partial G^{*}\left(x, \omega, x^{\prime}\right)}{\partial x}\right) .
\end{aligned}
$$

The quantity $\rho_{\mathrm{IF}}\left(x, \omega, x^{\prime}\right)$ at field point $x$ essentially describes the contributions of the left and right propagating fields originating from the source point $x^{\prime}$. The term $\rho_{\mathrm{IF}}\left(x, \omega, x^{\prime}\right)$ fully accounts for the reflections, losses, and interference and is closely related to the concepts of LDOS and NLDOS. Therefore, we refer to it as the interference density of states (IFDOS). In contrast to the NLDOS, the integral of the IFDOS with respect to $x^{\prime}$ is always zero as required, e.g., by the fact that in a medium in equilibrium, there is no net power flow, i.e., $\langle\hat{S}(x, t)\rangle_{\omega}=0$ when $\left\langle\hat{\eta}\left(x^{\prime}, \omega\right)\right\rangle$ is constant.

\section{Left and right propagating fields}

To generalize the LDOS and photon-number concepts in Refs. [3] and [4] and Eq. (1) to separately account for the left and right propagating fields, we write the left and right propagating field Poynting vector expectation values $\left\langle\hat{S}_{+}(x, \omega)\right\rangle$ and $\left\langle\hat{S}_{-}(x, \omega)\right\rangle$ as $\left\langle\hat{S}_{ \pm}(x, \omega)\right\rangle_{\omega}=\hbar \omega v(x, \omega) \rho_{ \pm}(x, \omega)\left(\left\langle\hat{n}_{ \pm}(x, \omega)\right\rangle+\right.$ $\left.\frac{1}{2}\right)$, where $\rho_{ \pm}(x, \omega)$ and $\left\langle\hat{n}_{ \pm}(x, \omega)\right\rangle$ are the left and right propagating field LDOSs and photon numbers to be determined, and the term one half describes the zero-point fluctuation current. The left and right propagating photon numbers must additionally satisfy two equations: the total Poynting vector must be given by

$$
\begin{aligned}
\langle\hat{S}(x, t)\rangle_{\omega}= & \hbar \omega v(x, \omega) \rho_{+}(x, \omega)\left(\left\langle\hat{n}_{+}(x, \omega)\right\rangle+\frac{1}{2}\right) \\
& -\hbar \omega v(x, \omega) \rho_{-}(x, \omega)\left(\left\langle\hat{n}_{-}(x, \omega)\right\rangle+\frac{1}{2}\right),
\end{aligned}
$$

and the total energy density $\langle\hat{u}(x, t)\rangle_{\omega}=$ $\hbar \omega \rho(x, \omega)\left(\langle\hat{n}(x, \omega)\rangle+\frac{1}{2}\right)[4]$ by

$$
\begin{aligned}
\langle\hat{u}(x, t)\rangle_{\omega}= & \hbar \omega \rho_{+}(x, \omega)\left(\left\langle\hat{n}_{+}(x, \omega)\right\rangle+\frac{1}{2}\right) \\
& +\hbar \omega \rho_{-}(x, \omega)\left(\left\langle\hat{n}_{-}(x, \omega)\right\rangle+\frac{1}{2}\right) .
\end{aligned}
$$

At zero temperature, where $\left\langle\hat{n}_{+}(x, \omega)\right\rangle=\left\langle\hat{n}_{-}(x, \omega)\right\rangle=0$, the Poynting vector is zero and thus $\rho_{+}(x, \omega)=\rho_{-}(x, \omega)$ in Eq. (6). Respectively, Eq. (7) at zero temperature leads to the relation $\rho_{+}(x, \omega)+\rho_{-}(x, \omega)=\rho(x, \omega)$. Together, these conditions uniquely define the left and right propagating LDOSs in terms of the total LDOS as $\rho_{+}(x, \omega)=\rho_{-}(x, \omega)=\rho(x, \omega) / 2$.

Using the above local density of states relations, we can uniquely solve the left and right propagating photon numbers from Eqs. (6) and (7) as $\left\langle\hat{n}_{ \pm}(x, \omega)\right\rangle=$ $[\hbar \omega \rho(x, \omega)]^{-1}\left[\langle\hat{u}(x, t)\rangle_{\omega} \pm\langle\hat{S}(x, t)\rangle_{\omega} / v(x, \omega)\right]-1 / 2$. In terms 
of the source field photon number this corresponds to

$$
\left\langle\hat{n}_{ \pm}(x, \omega)\right\rangle=\frac{\int_{-\infty}^{\infty}\left[\rho_{\mathrm{NL}}\left(x, \omega, x^{\prime}\right) \pm \rho_{\mathrm{IF}}\left(x, \omega, x^{\prime}\right)\right]\left\langle\hat{\eta}\left(x^{\prime}, \omega\right)\right\rangle d x^{\prime}}{\int_{-\infty}^{\infty}\left[\rho_{\mathrm{NL}}\left(x, \omega, x^{\prime}\right) \pm \rho_{\mathrm{IF}}\left(x, \omega, x^{\prime}\right)\right] d x^{\prime}} .
$$

Equation (8) shows that the propagating field photon-number expectation values are also obtained as a weighted sum of the source field values, but the weight factor now includes an additional term describing the interference and propagation direction. In the denominator, one can neglect $\rho_{\mathrm{IF}}\left(x, \omega, x^{\prime}\right)$ as it integrates to zero indicating that the denominator is simply equal to the LDOS in Eq. (3).

Above we have only focused on the photon-number expectation values that can be directly extracted from the Poynting vector. To find the corresponding ladder and photon-number operators in the QFED we will further investigate the forms of the photon annihilation operators $\hat{a}_{+}(x, \omega)$ and $\hat{a}_{-}(x, \omega)$ that lead to the expectation values in Eq. (8) and fulfill the canonical commutation relations. The photon annihilation operators fulfilling these conditions are of the form

$$
\begin{aligned}
\hat{a}_{ \pm}(x, \omega)= & \frac{1}{\sqrt{\rho(x, \omega)}} \int_{-\infty}^{\infty} e^{i(\phi \pm \pi / 4)} \\
& \times \sqrt{\rho_{\mathrm{NL}}\left(x, \omega, x^{\prime}\right) \pm \rho_{\mathrm{IF}}\left(x, \omega, x^{\prime}\right)} \hat{f}\left(x^{\prime}, \omega\right) d x^{\prime},
\end{aligned}
$$

where $\hat{f}\left(x^{\prime}, \omega\right)$ is a bosonic source field operator obeying the canonical commutation relation $\left[\hat{f}(x, \omega), \hat{f}^{\dagger}\left(x^{\prime}, \omega^{\prime}\right)\right]=$ $\delta\left(x-x^{\prime}\right) \delta\left(\omega-\omega^{\prime}\right)$ and which is related to the source field photon number as $\left\langle\hat{\eta}\left(x^{\prime}, \omega\right)\right\rangle=\int\left\langle\hat{f}^{\dagger}\left(x^{\prime}, \omega\right) \hat{f}\left(x^{\prime \prime}, \omega^{\prime}\right)\right\rangle d x^{\prime \prime} d \omega^{\prime}$ $[3,4,10]$. The phase factor $\phi$ is in principle arbitrary and it does not play a role in our calculations as it cancels in the commutators. The total field annihilation operator $\hat{a}(x, \omega)$ is given by the sum $\hat{a}(x, \omega)=\frac{1}{\sqrt{2}}\left[\hat{a}_{+}(x, \omega)+\hat{a}_{-}(x, \omega)\right]$. It is straightforward to check that the left and right propagating field annihilation operators in Eq. (9) also obey the commutation relation of the form $\left[\hat{a}_{ \pm}(x, \omega), \hat{a}_{ \pm}^{\dagger}\left(x, \omega^{\prime}\right)\right]=\delta\left(\omega-\omega^{\prime}\right)$. With these choices, however, the cross-commutators become nonzero as $\left[\hat{a}_{ \pm}(x, \omega), \hat{a}_{\mp}^{\dagger}\left(x, \omega^{\prime}\right)\right] \neq 0$ due to the coupling of the left and right propagating fields originating from the same source points by the reflecting interfaces. This crosscommutator form is intuitively reasonable and does not appear to present any complications as the only commutation relations directly linked to the studied physical observables are the self-commutators.

As shown by Eqs. (8) and (9) it is necessary to separately account for all the individual source points and their mutual interference to arrive to the correctly commutating operator forms. Similar bookkeeping is also present in classical fluctuational electrodynamics (FED). In contrast to the FED, however, to describe the quantum features the photon ladder and number operators need to be renormalized to fully satisfy the commutation relations.

In addition to describing the total energy density and energy flow presented in Eqs. (4), (6), and (7), the QFED formalism is also capable of separating the total field photon numbers to their local electric- and magnetic-field equivalents $\left\langle\hat{n}_{\mathrm{e}}(x, \omega)\right\rangle$ and $\left\langle\hat{n}_{\mathrm{m}}(x, \omega)\right\rangle$ that are responsible for direct interactions with materials and determine, e.g., the self-consistent local temperature of the interacting media as discussed in Refs. [4] and [3]. Essentially these electric- or magnetic-field specific quantities and the corresponding LDOSs can be obtained by using Eqs. (1) and (3) when only the electric- or magneticfield term in the NLDOS in Eq. (2) is taken into account [4]. The electric- and magnetic-field specific quantities were previously shown to have quite distinct properties as compared to the total field quantities and to include, e.g., oscillations in the field temperatures $[3,4]$. In the propagating operator formalism, however, the electric- and magnetic-field specific ladder operators are again united with the total propagating field operators because the direct interference effects between the left and right propagating fields have been eliminated when projecting the ladder operators to the left and right propagating operators. This fully agrees with our previous results $[3,4]$ : in the present formalism the separation to left and right propagating fields also fully separates the interference effects from the local fields, whereas the formalism simultaneously capturing both left and right propagating fields in a single term must also capture the interference effects.

\section{RESULTS}

To better illustrate the physical implications of the presented concepts we briefly discuss the properties of photon numbers of the left and right propagating fields and compare them to the corresponding total field photon number in an optical cavity consisting of three homogeneous layers as illustrated in Fig. 1.

\section{A. Lossless cavity structure}

In a lossless configuration, the left and right propagating field photon numbers are piecewise continuous and only depend on the cavity geometry and the input fields $\left\langle\hat{n}_{1+}\right\rangle$ and $\left\langle\hat{n}_{3-}\right\rangle$ incident from the left and right. In different regions, they can be written as

$$
\begin{aligned}
& \left\langle\hat{n}_{1-}\right\rangle=\left|\mathcal{R}_{1}\right|^{2}\left\langle\hat{n}_{1+}\right\rangle+\sqrt{\varepsilon_{1} / \varepsilon_{3}}\left|\mathcal{T}_{1}^{\prime} \mathcal{T}_{2}^{\prime}\right|^{2}\left\langle\hat{n}_{3-}\right\rangle, \\
& \left\langle\hat{n}_{2+}\right\rangle=\frac{\sqrt{\varepsilon_{2} / \varepsilon_{1}}\left|\mathcal{T}_{1}\right|^{2}\left\langle\hat{n}_{1+}\right\rangle+\sqrt{\varepsilon_{2} / \varepsilon_{3}}\left|\mathcal{T}_{2}^{\prime} \mathcal{R}_{1}^{\prime}\right|^{2}\left\langle\hat{n}_{3-}\right\rangle}{\operatorname{Re}\left[1+2 \mathcal{R}_{1}^{\prime} \mathcal{R}_{2} \nu_{2} e^{2 i k_{2} d_{2}}\right]} \\
& \left\langle\hat{n}_{2-}\right\rangle=\frac{\sqrt{\varepsilon_{2} / \varepsilon_{1}}\left|\mathcal{T}_{1} \mathcal{R}_{2}\right|^{2}\left\langle\hat{n}_{1+}\right\rangle+\sqrt{\varepsilon_{2} / \varepsilon_{3}}\left|\mathcal{T}_{2}^{\prime}\right|^{2}\left\langle\hat{n}_{3-}\right\rangle}{\operatorname{Re}\left[1+2 \mathcal{R}_{1}^{\prime} \mathcal{R}_{2} \nu_{2} e^{2 i k_{2} d_{2}}\right]}, \\
& \left\langle\hat{n}_{3+}\right\rangle=\sqrt{\varepsilon_{3} / \varepsilon_{1}}\left|\mathcal{T}_{1} \mathcal{T}_{2}\right|^{2}\left\langle\hat{n}_{1+}\right\rangle+\left|\mathcal{R}_{2}^{\prime}\right|^{2}\left\langle\hat{n}_{3-}\right\rangle
\end{aligned}
$$

where $d_{2}$ is the cavity thickness, $k_{2}$ is the wave number inside the cavity, $v_{2}=1 /\left(1+r_{1} r_{2} e^{2 i k_{2} d_{2}}\right), \mathcal{R}_{1}=\left(r_{1}+\right.$ $\left.r_{2} e^{2 i k_{2} d_{2}}\right) v_{2}, \quad \mathcal{R}_{2}=r_{2}, \mathcal{T}_{1}=t_{1} v_{2}, \mathcal{T}_{2}=t_{2}, \quad \mathcal{R}_{1}^{\prime}=r_{1}^{\prime}, \quad \mathcal{R}_{2}^{\prime}=$

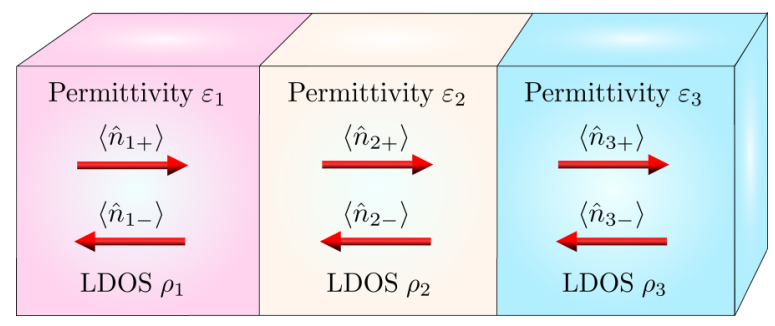

FIG. 1. (Color online) Optical cavity consisting of three homogeneous layers. We calculate the left and right propagating field photon-number expectation values in each layer. 


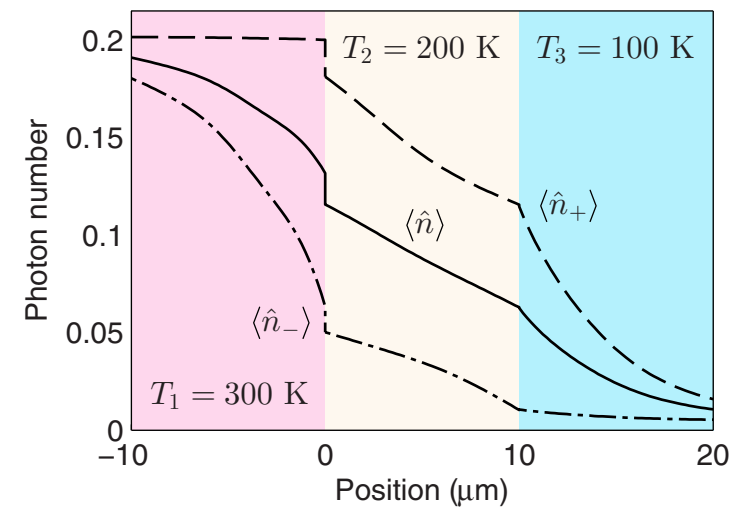

FIG. 2. (Color online) Left and right propagating photon numbers $\left\langle\hat{n}_{-}\right\rangle$and $\left\langle\hat{n}_{+}\right\rangle$and the total photon number $\langle\hat{n}\rangle$ in a lossy cavity structure at the first resonant energy $\hbar \omega=0.046 \mathrm{eV}(\lambda=$ $26.9 \mu \mathrm{m})$. The media from left to right have refractive indices $\sqrt{\varepsilon_{1}}=2.5+0.4 i, \sqrt{\varepsilon_{2}}=1.2+0.2 i$, and $\sqrt{\varepsilon_{3}}=1.5+0.5 i$, and source field temperatures $T_{1}=300 \mathrm{~K}, T_{2}=200 \mathrm{~K}$, and $T_{3}=100 \mathrm{~K}$.

$\left(r_{2}^{\prime}+r_{1}^{\prime} e^{2 i k_{2} d_{2}}\right) \nu_{2}, \mathcal{T}_{1}^{\prime}=t_{1}^{\prime}$, and $\mathcal{T}_{2}^{\prime}=t_{2}^{\prime} \nu_{2}$ with the conventional single interface Fresnel reflection and transmission coefficients for left incidence $r_{i}$ and $t_{i}, i \in\{1,2\}$, and right incidence $r_{i}^{\prime}$ and $t_{i}^{\prime}, i \in\{1,2\}$. In contrast, e.g., to the electricfield values where resonance effects can substantially increase the field magnitude inside a resonator, the photon-number values inside the cavity and at the outputs in Eq. (10) are always between the input field photon numbers. This also ensures that in global thermal equilibrium all the photon numbers are equal and no photon-number accumulation can occur inside the cavity.

\section{B. Lossy cavity structure}

In a lossy structure the photon numbers are no longer piecewise constant and all material points can act as field sources through the source field $\left\langle\hat{\eta}\left(x^{\prime}, \omega\right)\right\rangle$, which is related to material temperature. To illustrate this, we study a lossy cavity structure, where the refractive indices of the media are $\sqrt{\varepsilon_{1}}=2.5+0.4 i, \sqrt{\varepsilon_{2}}=1.2+0.2 i$, and $\sqrt{\varepsilon_{3}}=1.5+0.5 i$, and the layer temperatures are $T_{1}=300 \mathrm{~K}, T_{2}=200 \mathrm{~K}$, and $T_{3}=100 \mathrm{~K}$. Here the layer temperatures are set to constant predefined values for simplicity even if the QFED formalism also allows calculating the in-cavity temperature distribution self-consistently if the studied layers were to be considered as thermal insulators $[4,10]$. Figure 2 shows the total, right propagating, and left propagating photon numbers $\langle\hat{n}\rangle,\left\langle\hat{n}_{+}\right\rangle$, and $\left\langle\hat{n}_{-}\right\rangle$as a function of position at the first cavity resonance $\hbar \omega=0.046 \mathrm{eV}(\lambda=26.9 \mu \mathrm{m})$, where the layer temperatures correspond to steady-state photon numbers $0.20,0.074$, and 0.0048 . The photon numbers are highest at the leftmost medium at $T_{1}=300 \mathrm{~K}$ and decrease towards the rightmost medium at $T_{3}=100 \mathrm{~K}$. The right propagating photon number notably decreases at and after the first interface due to reflection and thermalization, eventually reaching equilibrium with the lossy medium in the rightmost layer. The left propagating photon number notably changes at the interfaces and in the middle and leftmost layers. It can be also clearly seen that the total photon number is the average of the left and right propagating photon numbers as expected, since the photon number essentially describes the average photon number in the collection of optical modes under study.

As the photon-number expectation value depends strongly on the frequency, it is convenient to illustrate the results by using the effective field temperature that is defined in terms of the photon-number expectation value as $T_{\text {eff }}(x, \omega)=$ $\hbar \omega /\left\{k_{\mathrm{B}} \ln [1+1 /\langle\hat{n}(x, \omega)\rangle]\right\}[4,10]$. This corresponds to the steady-state temperature of a small temperature probe interacting only with a single mode [4]. Figure 3 shows the total LDOS and field temperatures corresponding to the total, right propagating, and left propagating fields as a function of position and photon energy. In contrast to the case of a lossless structure, the field quantities are position dependent. The total LDOS in Fig. 3(a) is also oscillatory inside the cavity and reaches its maxima at resonant energies $\hbar \omega=0.046 \mathrm{eV}(\lambda=$ $26.9 \mu \mathrm{m}), \hbar \omega=0.097 \mathrm{eV}(\lambda=12.7 \mu \mathrm{m})$, and $\hbar \omega=0.150 \mathrm{eV}$ $(\lambda=8.29 \mu \mathrm{m})$. Also in the left- and rightmost layers, the total LDOS is position dependent and oscillatory near interfaces. The oscillations of the LDOS follow from the interference effects combined with the material polarizability in analogy with the Purcell effect [4].

Despite the oscillations in the LDOS, the total effective field temperature $T_{\text {eff }}$ in Fig. 3(b) and the effective field temperatures $T_{\mathrm{eff}}^{+}$and $T_{\mathrm{eff}}^{-}$corresponding to the right and left propagating fields in Figs. 3(c) and 3(d) decrease towards the right medium at lower temperature similar to the photon numbers in Fig. 2. On the left and right $T_{\text {eff }}, T_{\text {eff }}^{+}$, and $T_{\text {eff }}^{-}$also asymptotically approach equilibrium values corresponding to material temperatures. When compared to $T_{\text {eff }}^{+}$, the magnitude of $T_{\text {eff }}^{-}$is everywhere lower since the source field temperature on the right is lower than the source field temperature on the left.

\section{CONCLUSIONS}

In conclusion, we have developed a generalized quantum optical noise formalism QFED that can unambiguously describe the quantum aspects of propagating optical fields in arbitrary stratified media, while being fully compliant with the canonical commutation relations. In particular, the QFED allows calculating position-dependent photon-number expectation values for the left and right propagating fields and fully eliminates the anomalies of the ladder operators in optical cavities. In our model, the commutation relations are therefore canonical. This implies that, in contrast to previous models that involve anomalous commutation relations, our results do not predict, e.g., any observable threshold for the second harmonic generation inside cavities as the threshold is directly linked to the ladder operators. Experimental measurements of the existence of a second harmonic generation threshold may therefore allow demonstrating the importance of correct normalization of the commutation relations as well as the affiliated normal modes. In addition, the QFED framework enables, e.g., the separation of the quantum optical Poynting vector and related field quantities to their left and right propagating components using a photon-number-based presentation and the interference density of states. In practical modeling tasks, 

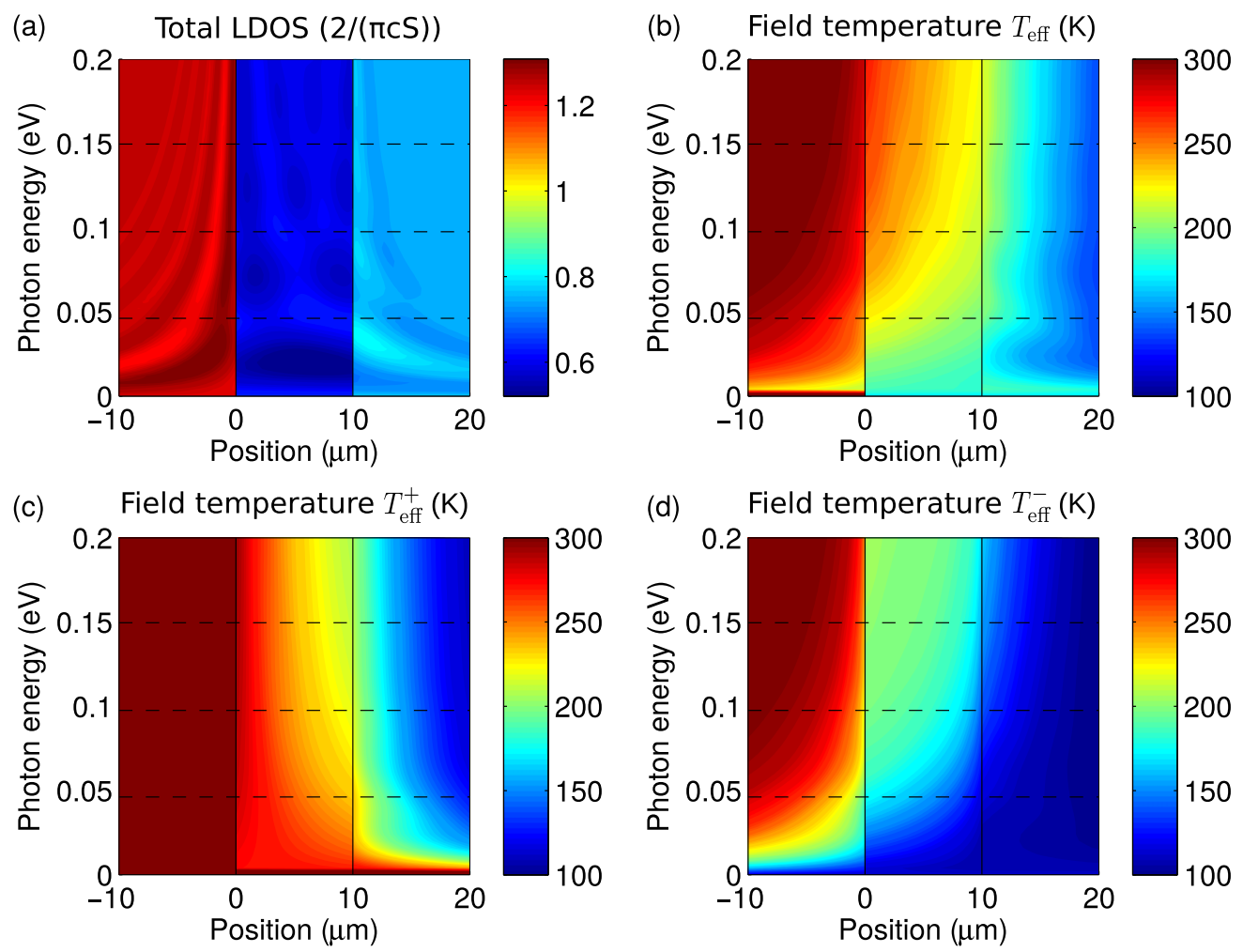

FIG. 3. (Color online) (a) Total LDOS, (b) $T_{\text {eff }}$, (c) $T_{\text {eff }}^{+}$, and (d) $T_{\text {eff }}^{-}$in a lossy cavity structure. The media from left to right have refractive indices $\sqrt{\varepsilon_{1}}=2.5+0.4 i, \sqrt{\varepsilon_{2}}=1.2+0.2 i$, and $\sqrt{\varepsilon_{3}}=1.5+0.5 i$, and layer temperatures $T_{1}=300 \mathrm{~K}, T_{2}=200 \mathrm{~K}$, and $T_{3}=100 \mathrm{~K}$. Solid lines denote the boundaries of the cavity and dashed lines denote resonant energies. The LDOS is given in the units of $2 /(\pi c S)$.

the QFED provides simple tools for studying optical energy transfer and the formation of thermal balance in complex interfering nanostructures, highlighting the fundamentally nonlocal nature of the energy transfer.

\section{ACKNOWLEDGMENT}

This work has in part been funded by the Academy of Finland and the Aalto Energy Efficiency Research Programme.
[1] S. Gauvin, M. Collette, and N. Beaudoin, Second harmonic generation inside microcavities: On the existence of a threshold, in Frontiers in Optics (OSA, Washington, DC, 2014).

[2] M. Collette, N. Beaudoin, and S. Gauvin, Second order optical nonlinear processes as tools to probe anomalies inside high confinement microcavities, Proc. SPIE 8772, 87721D (2013).

[3] M. Partanen, T. Häyrynen, J. Oksanen, and J. Tulkki, Thermal balance and photon-number quantization in layered structures, Phys. Rev. A 89, 033831 (2014).

[4] M. Partanen, T. Häyrynen, J. Oksanen, and J. Tulkki, Unified position-dependent photon-number quantization in layered structures, Phys. Rev. A 90, 063804 (2014).

[5] M. Ueda and N. Imoto, Anomalous commutation relation and modified spontaneous emission inside a microcavity, Phys. Rev. A 50, 89 (1994).

[6] M. G. Raymer and C. J. McKinstrie, Quantum input-output theory for optical cavities with arbitrary coupling strength: Application to two-photon wave-packet shaping, Phys. Rev. A 88, 043819 (2013).

[7] S. M. Barnett, C. R. Gilson, B. Huttner, and N. Imoto, Field Commutation Relations in Optical Cavities, Phys. Rev. Lett. 77, 1739 (1996).
[8] A. Aiello, Input-output relations in optical cavities: A simple point of view, Phys. Rev. A 62, 063813 (2000).

[9] O. Di Stefano, S. Savasta, and R. Girlanda, Three-dimensional electromagnetic field quantization in absorbing and dispersive bounded dielectrics, Phys. Rev. A 61, 023803 (2000).

[10] M. Partanen, T. Häyrynen, J. Oksanen, and J. Tulkki, Positiondependent photon operators in the quantization of the electromagnetic field in dielectrics at local thermal equilibrium, in Proc. SPIE 9136, Nonlinear Optics and Its Applications VIII; and Quantum Optics III, No. 91362B (SPIE, Bellingham, WA, 2014).

[11] L. Knöll, W. Vogel, and D. G. Welsch, Action of passive, lossless optical systems in quantum optics, Phys. Rev. A 36, 3803 (1987).

[12] L. Knöll, W. Vogel, and D.-G. Welsch, Resonators in quantum optics: A first-principles approach, Phys. Rev. A 43, 543 (1991).

[13] L. Allen and S. Stenholm, Quantum effects at a dielectric interface, Opt. Commun. 93, 253 (1992).

[14] B. Huttner and S. M. Barnett, Quantization of the electromagnetic field in dielectrics, Phys. Rev. A 46, 4306 (1992).

[15] S. M. Barnett, R. Matloob, and R. Loudon, Quantum theory of a dielectric-vacuum interface in one dimension, J. Mod. Opt. 42, 1165 (1995). 
[16] R. Matloob, R. Loudon, S. M. Barnett, and J. Jeffers, Electromagnetic field quantization in absorbing dielectrics, Phys. Rev. A 52, 4823 (1995).

[17] R. Matloob and R. Loudon, Electromagnetic field quantization in absorbing dielectrics. II, Phys. Rev. A 53, 4567 (1996).
[18] M. Janowicz, D. Reddig, and M. Holthaus, Quantum approach to electromagnetic energy transfer between two dielectric bodies, Phys. Rev. A 68, 043823 (2003).

[19] R. Loudon, The Quantum Theory of Light (Oxford University Press, Oxford, 2000). 